\title{
Assessment of potential Al ion conductors from large crystallographic databases
}

Falk Meutzner $^{1}$, Tina Nestler ${ }^{1}$, Matthias Zschornak ${ }^{1}$, Artem Kabanov², Tilmann Leisegang ${ }^{1,2}$, Vladislav A. Blatov ${ }^{2}$, Dirk C. Meyer ${ }^{1}$

${ }^{1}$ TU Bergakademie Freiberg, Freiberg, Germany, ${ }^{2}$ Samara University, Samara, Russian Federation E-mail: falk.meutzner@physik.tu-freiberg.de

The possibility to store electrical energy enables not only mobile electronics and electric cars, but will be of paramount importance for the advancing penetration of renewables in the energy grid. Due to the capricious nature of sustainable energy carriers, in times of high production, energy will be stored for times of low or no production in order to balance out offer and demand. $\mathrm{Li}$ and $\mathrm{Pb}$ strongly dominate the battery market at the moment but the search for new chemistries and technologies for a diversification of applications will become increasingly important in the future. Aluminium offers a substantially higher abundancy, even higher theoretical energy densities, and may profit from its technological maturity. It is therefore a highly-promising candidate for post-Li chemistries.

To this point, materials for secondary Al based electrochemical energy storage devices - i.e. electrodes and solid electrolytes - are not known or have not been proven unambiguously regarding their functionality. This is also due to the high charge of the trivalent Al ion, which is supposed to strongly impede an ionic conduction. Furthermore, finding new materials has been a very time-consuming process based on trial-and-error or step-by-step modification of materials already known for their ionic diffusion behaviour.

From a crystallographic point of view, cation diffusion or conduction in crystalline materials can be described as jumps of a mobile cation between interconnected crystallographic sites in anion-coordinated voids. Based on this theory, VoronoiDirichlet partitioning can quickly determine structure-immanent void-systems of large crystal structure databases (e.g. the ICSD). The results can be filtered using a range of crystallographic and economic parameters to point towards the highestpromise materials [1]. Afterwards, bond-valence methodology verifies the results and estimates activation energies in order to rank the results obtained beforehand. Eventually, the most promising materials are analysed with density functional theory in order to simulate a full, dynamic diffusion process, deducing the most important electrochemical parameters.

This work presents the screening for Al ion conductors, involving all three approaches and their comparison. The results for oxygen-containing compounds will be discussed and a comparison and outlook for sulphur-containing materials will be given. This work is financially supported by the German BMBF (R2R Battery: 03SF0542A; CryPhysConcept: 03EK3029A), the Russian Megagrant (14.B25.31.0005), and the RSF (16-13-10158).

[1] Meutzner, F. \& Münchgesang, W. \& Leisegang, T. \& Schmid, R. \& Zschornak, M. \& Ureña de Vivanco, M. \& Shevchenko; A. P. \& Blatov, V. A. \& Meyer, D. C. (2017). Crystal Research \& Technology, 52, 1600223.

Keywords: solid electrolytes, Al batteries, electrochemistry 